\title{
Development of a literature informed Bayesian machine learning method for feature extraction and classification
}

\author{
Behrouz Madahian', Lih Yuan Deng ${ }^{1}$, Ramin Homayouni ${ }^{2,3 *}$ \\ From 14th Annual UT-KBRIN Bioinformatics Summit 2015 \\ Buchanan, TN, USA. 20-22 March 2015
}

\section{Background}

Gene expression profiling is a powerful approach to identify markers for classification of samples; however, it has major limitations that hinder performance. Typically, a large number of variables are assessed compared to relatively small sample sizes. In addition, it is difficult to identify biologically informative markers which have high predictive power [1-3]. Thus, the goal of this study was to develop a machine learning approach that is able to bridge classification accuracy and biological function.

\section{Materials and methods}

We developed a Literature aided Sparse Bayesian Generalized Linear model which utilizes Generalized Double Pareto (LSBGG) prior to induce shrinkage in terms of the number of covariates. Importantly, instead of using uninformed hyper parameters for the prior distributions, we adjusted the hyper parameters based on the ranking of the genes by GeneIndexer (Quire Inc. Memphis, TN) with respect to 'cancer' keyword query. This unique approach controls shrinkage imposed on genes based on biological function extracted from the literature. The model was applied to a leukemia data set from Golub et al. [4]. The dataset was split into training and test groups and classification performance was evaluated on the test group. The top 500 highly differentially expressed genes were used for the modeling step.

\section{Results}

Using the top 10 genes obtained from LSBGG, we were able to achieve $91 \%$ classification accuracy in the test group. When the training and test datasets were switched,

\footnotetext{
* Correspondence: rhomayoun@memphis.edu

${ }^{2}$ Bioinformatics Program, University of Memphis, Memphis, TN 38152, USA Full list of author information is available at the end of the article
}

we obtained $92 \%$ classification accuracy. In contrast, the model without biological information achieved $91 \%$ and $86 \%$ classification accuracies in the two test scenarios (Table 1). Consistent with these results, Receiver Operating Characteristic (ROC) analysis showed better performance when shrinkage was imposed using the literature (Figure 1). Notably, we found that the posterior mean of $\theta$

Table 1 Classification accuracy, sensitivity and specificity of the model including (LSBGG) or excluding literature.

\begin{tabular}{lcccc}
\hline & \multicolumn{2}{c}{ Including Literature } & \multicolumn{2}{c}{ Excluding Literature } \\
\cline { 2 - 5 } & Test1 & Test2 & Test3 & Test4 \\
Measure & & & & \\
\hline Accuracy & 91 & 92 & 91 & 86 \\
Sensitivity & 100 & 89 & 100 & 91 \\
Specificity & 79 & 100 & 75 & 75 \\
\hline
\end{tabular}

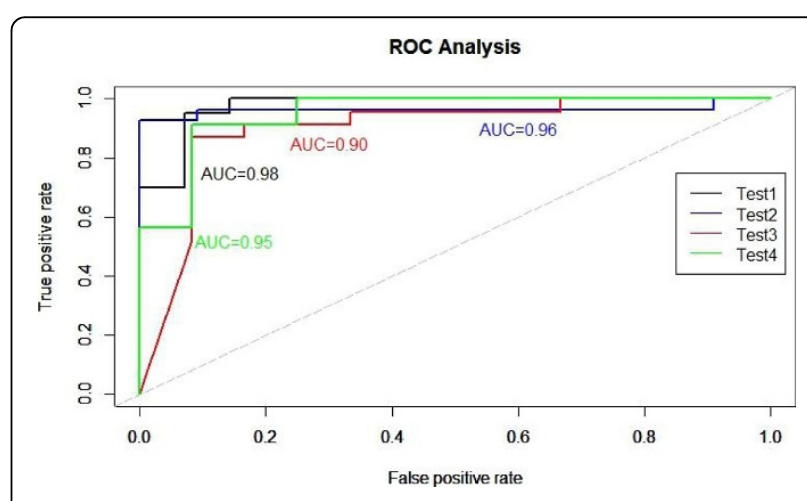

Figure 1 ROC curves for the models with or without literature imposed shrinkage. The area under the curve (AUC) for the model incorporating literature (Tests 1 \&2) is higher than the model without incorporating literature (Tests 3 \&4). 
(A)

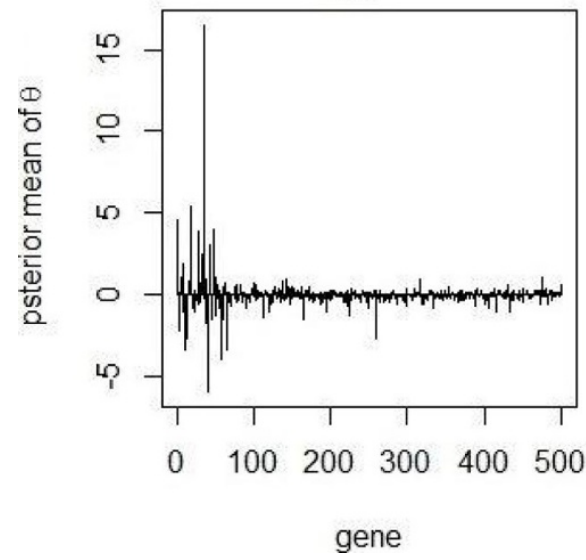

(B)

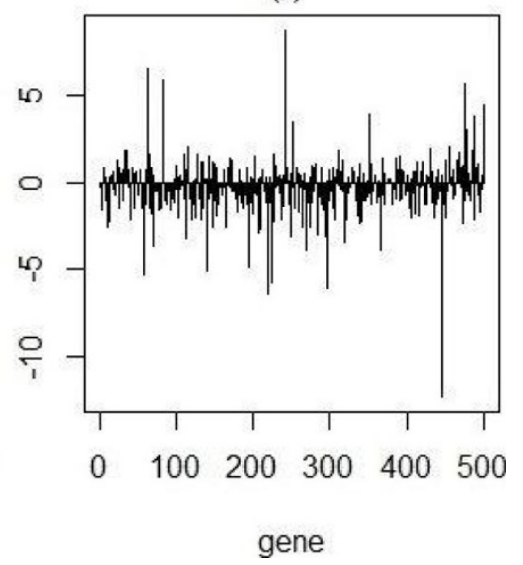

Figure 2 Relationship between the posterior mean of $\theta$ s and biological relevance. The posterior mean of $\theta$ (Y-axis) is shown for the top 500 genes (X-axis) when using a model with (A) or without (B) incorporation of literature to control shrinkage. There is a clear association between the estimated $\theta$ in the model and association with cancer in the literature.

was higher for genes which were functionally related to cancer in the biomedical literature (Figure 2).

\section{Conclusions}

This demonstrates that while LBSGG performs slightly better in classification of samples, it uses more biologically informative genes, and hence may simultaneously provide insights into the mechanisms underlying the phenotype of interest.

\section{Acknowledgements}

This work was supported by the University of Memphis Center for

Translational Informatics and the Assisi Foundation of Memphis.

\section{Authors' details}

'Department of Mathematical Sciences, University of Memphis, Memphis, TN 38152, USA. ${ }^{2}$ Bioinformatics Program, University of Memphis, Memphis, TN 38152, USA. ${ }^{3}$ Department of Biological Sciences, University of Memphis, Memphis, TN 38152, USA.

Published: 23 October 2015

\section{References}

1. Dupuy A, Simmon RM: Critical Review of Published Microarray Studies for Cancer Outcome and Guidelines on Statistical Analysis and Reporting. JNCI J Natl Cancer Inst 2007, 2:147-157.

2. Madahian B, Deng L, Homayouni R: Application of Sparse Bayesian Generalized Linear Model to Gene Expression Data for Classification of Prostate Cancer Subtypes. Open Journal of Statistics 2014, 2:518-526.

3. Novianti PW, Roes KCB, Eijkemans MJC: Evaluation of Gene Expression Classification Studies: Factors Associated with Classification Performance. PLOS ONE 2014, 9(4):e96063.

4. Golub T, Slonim D, Tamayo P, Huard C, Gaasenbeek M, Mesirov J, Coller H, Loh M, Downing J, Caligiuri M, Bloomfield CE: Molecular classification of cancer: class discovery and class prediction by gene expression monitoring. Science 1999, 286:531-537.

doi:10.1186/1471-2105-16-S15-P9

Cite this article as: Madahian et al:: Development of a literature informed Bayesian machine learning method for feature extraction and classification. BMC Bioinformatics 2015 16(Suppl 15):P9.

\section{Submit your next manuscript to BioMed Central} and take full advantage of:

- Convenient online submission

- Thorough peer review

- No space constraints or color figure charges

- Immediate publication on acceptance

- Inclusion in PubMed, CAS, Scopus and Google Scholar

- Research which is freely available for redistribution 